\title{
VENA SAFENA MAGNANIN ANATOMIK VARYASYONU DERIN VEN TROMBOZU İÇİN BİR RİSK FAKTÖRÜ MÜDÜR?
}

\section{Is the Anatomic Variation of Vena Saphena Magna A Risk Factor for Deep Vein Thrombosis?}

\author{
Ali BOLAT ${ }^{1}$ iD, Yıldırım GÜLTEKİN ${ }^{1}$ \\ ${ }^{1}$ Kırlkkale Üniversitesi Tip Fakültesi, Kalp ve Damar Cerrahisi Anabilim Dall, KIRIKKALE, TÜRKIYE
}

Amaç: $\mathrm{Bu}$ retrospektif çalışmanın amacı, vena safena magnanın, anatomik varyasyonunun femoral ven trombozuna olan etkisini araştırmaktır.

Gereç ve Yöntemler: Bu çalışma Kırıkkale Üniversitesi Tıp Fakültesi Hastanesi Kalp ve Damar Cerrahisi Kliniğinde Ocak 2017 ve Ağustos 2019 tarihleri arasında takip ve tedavi edilen femoral ven trombozu tanis1 olan hastalarda yapild1. Hastaların demografik özellikleri, venöz renkli Doppler ultrasonografi raporları ve eşlik eden hastalıklar retrospektif olarak incelendi. Vena safena magnanın tipleri, uyluk seviyesinde safen kompartmanındaki yerleşimlerine göre belirlendi.

Bulgular: Çalışma ana ve superfisiyal femoral vende derin ven trombozu tanısı konulan 130 hasta üzerinde yapılmıştır. Hastaların 62'si kadın ve 68'i erkekti ve yaşları 16 ile 89 yıl arasında değişmekteydi. Ortalama yaşları ise $55.8 \pm 17.3$ yıldı. Derin ven trombozu risk faktörlerinden, en s1k ileri yaş (\%37), en az oral kontraseptif kullanımı (\%1.5) gözlendi. On hastada predispozan faktör saptanamadi. Ana ve superfisiyal femoral derin ven trombozu olan hastalarda vena safena magna tiplerinin görülme yüzdeleri normal popülasyona göre aynı bulunmuştur.

Sonuç: Çalışmada ana ve superfisiyal femoral derin ven trombozu olan hastalarda vena safena magna varyasyonlarının görülme oranının normal popülasyona göre benzer olduğu saptand 1 .

Anahtar Kelimeler: Vena safena magna, anatomik varyasyon, derin ven trombozu
Objective: The aim of this retrospective study is to investigate the relationship between the anatomical variation of the vena saphena magna and femoral vein thrombosis.

Material and Methods: This study was conducted in patients with femoral vein thrombosis who were followed up and treated between January 2017 and August 2019 in Kırıkkale University Faculty of Medicine Hospital Cardiovascular Surgery Clinic. The demographic characteristics of the patients, venous color Doppler ultrasonography reports and accompanying diseases were retrospectively analyzed. Vena saphena magna types were determined according to their position in the saphenous compartment at the thigh level.

Results: The study was conducted on 130 patients diagnosed with deep vein thrombosis in the common and superficial femoral vein. Sixty-two of the patients were female and 68 were male and their ages ranged from 16 to 89 years. Mean age was $55.8 \pm 17.3$ years. Among the DVT risk factors, old age (37\%) was the most common and use of oral contraceptives (1.5\%) was the least observed. No predisposing factor was found in 10 patients. The percentages of vena saphena magna types in patients with common and superficial femoral deep vein thrombosis were found to be the same compared to the normal population.

Conclusion: In the study, the incidence of vena saphena magna variations in patients with common and superficial femoral deep vein thrombosis was found to be similar compared to the normal population.

Keywords: Vena saphena magna, anatomical variation, deep vein thrombosis

\section{Yazışma Adresi / Correspondence: \\ Dr. Yıldırım GÜLTEKİN \\ Kırıkkale Üniversitesi Tıp Fakültesi, Kalp ve Damar Cerrahisi Anabilim Dalı, KIRIKKALE, TÜRKIYE \\ Tel / Phone: +90 5052230082 \\ E-posta / E-mail: dr.ygultekin@ hotmail.com \\ Kabul Tarihi / Accepted: 04.06.2021}




\section{GİRIȘ}

Derin ven trombozu (DVT), derin venlerde trombüs oluşumu ile ortaya çıkan bir hastalıktır (1). Venöz tromboembolizm sık görülen, önlenebilir bir halk sağlığı problemidir. Aynı zamanda hastanede en çok görülen önlenebilir mortalite nedenlerindendir (2). DVT büyük bir çoğunlukla ileri yaşlarda görülen bir hastalıktır. Özellikle erkeklerde ve 40 yaş sonrası daha sık görülmektedir (3).

Trombozun etiyopatogenezi; kan akımındaki değişiklikler (staz), damar duvar değişiklikleri ve koagülopatiden oluşan Virchow'un 1856'da öne sürdüğü triad ile açıklanmaktadır (4). Ekstremitelerin çeşitli nedenlerle hareketsiz kalması, venöz staza ve sonucunda DVT'ye neden olabilir (5). Venöz kapakçıklar ve baldır kaslarının kasılması ile venöz akım sağlanır. Uzun süreli seyahatler, ameliyat sonrası dönemlerdeki immobilizasyon, tümör, gebelik veya obeziteye bağlı venöz kompresyonlar, konjestif kalp yetmezlĭgi, akut miyokard enfarktüsü ve kardiyomiyopatiye bağlı sol kalp yetmezlikleri sebebiyle venöz dolaşım bozulur. Kanın damar endoteli ile uzun süre temas etmesi ile özellikle kan akımının yavaş ve türbülan akımın olduğu bölgelerde trombüs oluşumu için yatkınlık vardır (6). Bunların yanı sıra safenofemoral bileşke (SFB) düzeyinde vena safena magnada (VSM) oluşan reflü, sifon etkisi ile femoral vende de reflüye ve türbülan akıma yol açtığ gösterilmiştir (7). Ayrıca SFB'ye yakın ya da burayı tutan VSM flebiti de femoral vene uzanan trombüse neden olabilmektedir (8).

Venöz reflünün neden olduğu venöz hipertansiyon sonucunda oluşan venöz yetmezlik $\% 95$ 'den fazla oranda yüzeyel venlerde ya da perforan venlerde görülür. Venöz yetmezlik nadiren derin venlerde de görülebilir; bu durum daha çok yüzeyel ven yetmezliğine sekonderdir. Ancak derin ven yetmezliği bazı durumlarda şiddetlidir ve yüzeyel ven yetmezliğinden bağımsızdır (9).
Yüzeyel venlerden olan VSM, derin fasia ve safen fasyası ile sınırlanan safen kompartmanında seyreder. $\mathrm{Bu}$ kompartman uyluk bölgesinde ultrasonografi ile kolayca gösterilebilir ve görünümü itibarı ile "Mısırlı Gözü” olarak adlandırılır (10).

Safenöz kompartmanın VSM'yi çevreleyen destekleyici bir yap1 görevi gördüğü ve safenöz kompartman ile VSM ilişkisinin venöz reflü ve varis gelişimi (staz) riski ile ilişkili olduğu bildirilmiştir (11).

Superfisyial ven trombozu (SVT) gelişimi için farklı risk faktörleri arasında varisli damarlar, trombofili, oral kontrasepsiyon kullanımı, immobilizasyon, malignite, doğrudan travma veya tromboembolizm sıralanabilir ve DVT risk faktörleri benzerdir. SVT ile ilişkili olarak DVT gelişmesinin farklı mekanizmaları tartışılmıştır $(12,13)$.

$\mathrm{Bu}$ çalışmada VSM anatomik varyasyonları ile ana ve superfisiyal femoral ven trombozu arasında bir ilişki olup olmadığını araştırıp sonuçlarını paylaşmayı amaçladık.

\section{GEREÇ VE YÖNTEM}

Kırıkkale Üniversitesi Tıp Fakültesi Hastanesi Kalp ve Damar Cerrahisi Kliniğinde Ocak 2017 ve Ağustos 2019 tarihleri arasında takip ve tedavi edilen ve ana ve yüzeyel femoral DVT saptanan toplam 130 hastanın verileri retrospektif olarak değerlendirildi. Çalışma Helsinki Deklarasyonunda belirtilen ilkeler doğrultusunda yapıldı. Etik kurul onayı Kırıkkale Üniversitesi Girişimsel Olmayan Araştırmalar Etik Kurulundan alınmıştır (Tarih: 30.09.2020, sayı no: 2020.09.10).

$\mathrm{Bu}$ retrospektif çalışma için hastane kayıt sisteminden hasta dosyaları incelendi ve hastaların demografik özellikleri, venöz renkli Doppler ultrasonografi (RDUS) raporları ve eşlik eden hastalık verileri kaydedildi.

Venöz RDUS incelemelerinde, VSM varyasyonlarını tiplendirmek için, ana ve yüzeyel femoral ven ve safenofemoral kesişime (SFJ) yakın anatomik bölge olan uyluk 
bölgesinde, safenöz kompartmanın değerlendirilmesine dayanan Ricci ve Caggiati tarafından tanımlanan VSM sınıflandırma sistemi kullanıldı (14) (Şekil 1). Bu sınıflandırma sistemine göre; Tip A: VSM, safenöz kompartman içinde kendine eşlik eden büyük paralel kol olmadan uzanır, Tip B: Uyluk bölgesinde aynı safenöz kompartmanda iki paralel VSM bulunur (duplikasyon), Tip C: Uyluktaki safenöz kompartmanda tek VSM ve
VSM'ye bağlı subkütan büyük bir dal bulunur, Tip D: İki venöz yapı, VSM ve anterior aksesuar safenöz ven uylukta aynı anda iki ayrı safenöz kompartmanda bulunur. SFJ alanına girmeden hemen önce tek bir safenöz kompartmanda birleşirler, Tip E: Çeşitli seviyelerde proksimal safenöz kompartmanda subkutan uzantılarla devam eden tek VSM'yi içerir.

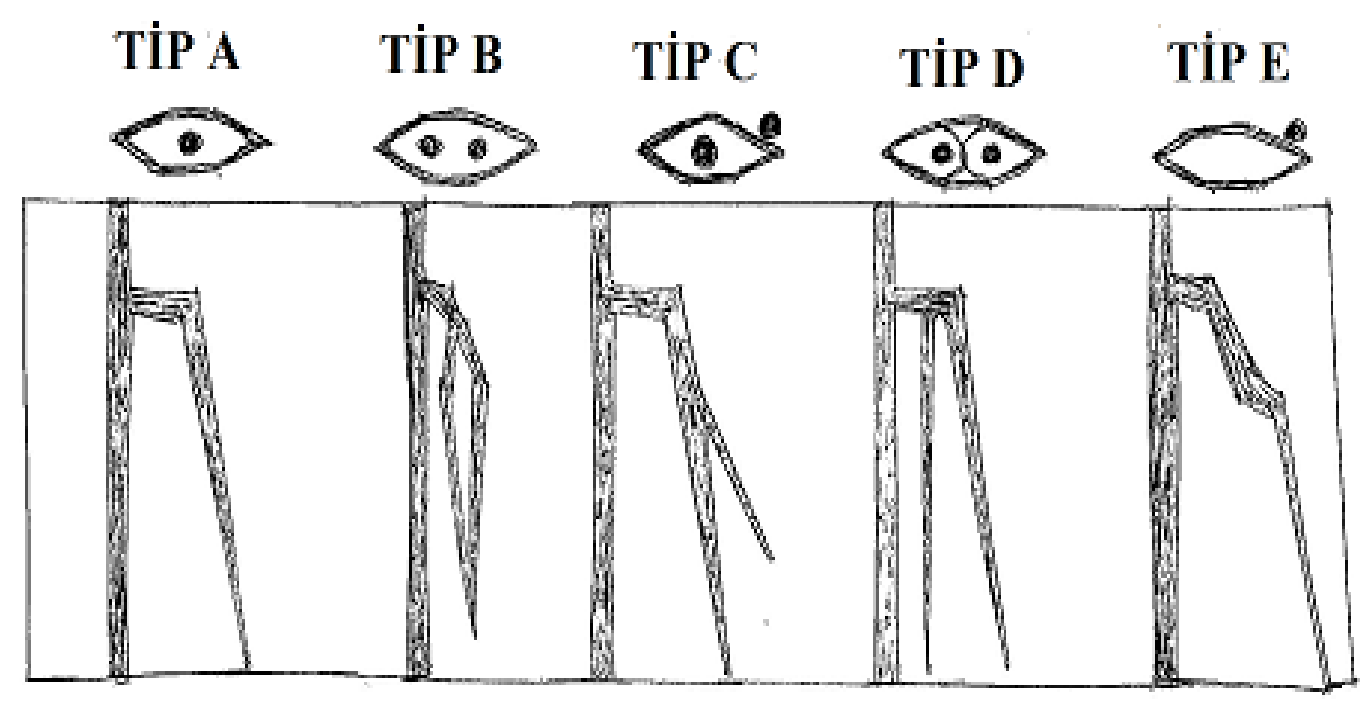

Şekil 1: Ricci ve Caggiati sınıflamasına göre vena safena magnanın anatomik varyasyonları

\section{Istatistiksel Analiz}

Çalışmada kullanılan verilerin analizi SPSS for Windows 24,0 paket programı (SPSS Inc. Chicago, IL, USA) kullanılarak yapıldı. Kategorik değişkenler yüzde, sürekli değişkenler ortalama \pm SD olarak ifade edildi. Çalışmada kullanılan verilerin normal dağılıma uygunluklarını değerlendirmek için KolmogorovSmirnov testi kullanıldı. Normal dağılıma uymayanlara non-parametrik testler, normal dağılıma uyanlara parametrik testler uyguland1.

\section{BULGULAR}

Hastaların 62'si (\%47.6) kadın ve 68'i (\%52.4) erkekti. Yaşları 16 ile 89 arasında değişmekteydi ve ortalama yaş ise $57.5 \pm 18.7$ yıldı. DVT risk faktörleri açısından incelendiğinde, en s1k ileri yaş (\%37) ve immobilizasyon (\%18.4) saptanmıştır. En az oral kontraseptif kullanımı (\%1.5) gözlenmiştir. Risk faktörleri Tablo 1'de gösterilmiştir. 
Çalışmada Ricci ve Caggiati sınıflamasına göre en sık Tip A (\%55.3) gözlenirken, en az sıklıkta Tip B (\%1.5) görülmüştür. Çalışmada tespit edilen safenöz kompartmana göre VSM varyasyonları Tablo 2'de verilmiştir.

Tablo 1: DVT risk faktörleri

\begin{tabular}{lcc}
\hline & $\begin{array}{c}\text { Sayı } \\
(\mathrm{n}=130)\end{array}$ & $\begin{array}{c}\text { Yüzde } \\
(\%)\end{array}$ \\
\hline İleri yaş & 48 & 37 \\
İmmobilizasyon & 24 & 18.5 \\
Obezite & 6 & 4.6 \\
Venöz tromboemboli öyküsü & 6 & 4.6 \\
İnflamatuar hastalıklar & 4 & 3.1 \\
Oral kontraseptif kullanımı & 2 & 1.5 \\
Kanser & 16 & 12.3 \\
Santral venöz kataterler & 4 & 3.1 \\
Faktör V Leiden mutasyonu & 6 & 4.6 \\
Risk Faktörü Saptanamayan & 10 & 7.6 \\
Variköz venler & 4 & 3.1 \\
\hline DVT: Derin ven trombozu & &
\end{tabular}

Tablo 2: VSM tip verileri

\begin{tabular}{lcc}
\hline & Sayı $(\mathrm{n}=130)$ & Yüzde $(\%)$ \\
\hline Tip A & 72 & 55.4 \\
Tip B & 2 & 1.5 \\
Tip C & 26 & 20 \\
Tip D & 8 & 6.2 \\
Tip E & 22 & 16.9 \\
\hline
\end{tabular}

VSM: Vena safena magna

\section{TARTIŞMA}

Alt veya üst ekstremite derin venlerinde oluşan, ayrıca visseral, serebral venlerde ya da vena kavada da meydana gelebilen tromboz, DVT olarak isimlendirilir.
Önlenebilir hastane ölümlerinde ilk sirada yer alan DVT, mortalitesi yüksek olan pulmoner emboliye neden olabilmesi nedeniyle önemli bir hastalıktır. Ayrıca tekrarlayan tromboz ve posttrombotik sendrom en önemli morbidite nedenleridir. Alt ekstremite derin venlerinde ve iliofemoral segmentin proksimal venlerinde daha sık görülür (15).

DVT insidansı genel popülasyonda yılda 10000 kişide 5 vaka olarak bildirilmiştir. İleri dekadlarda görülme ihtimali artmaktadır. Y1llık görülme sıklığı her 10.000 kişide 5-20 hasta arasında saptanmaktadır. Erkeklerde kadınlara oranla (1.2:1) daha fazla görülmektedir (16). Çalışmamızda da hastaların 62’si (\%47.6) kadın ve 68'i (\%52.4) erkek olarak saptand1. $\mathrm{Bu}$ literatürle uyumludur. Yaşları 16 ile 89 arasında değişmekteydi ve ortalama yaş ise $57.5 \pm 18.7$ y1l olarak bulundu.

DVT gelişimine neden olan üç temel patogenetik mekanizma gösterilmiştir. Kan akımının yavaşlaması (staz), damar duvarında hasar (endotel hasarı) ve hiperkoagülabilite, Virchow üçlüsü (triad1) olarak tanımlanmaktadır (17). İmmobilizasyonun da neden olduğu venöz staz DVTye neden olan en önemli risk faktörüdür (18).

$\mathrm{Bu}$ çalışmada hastalar, DVT risk faktörleri açısından incelendiğinde, en sık olarak ileri yaş (\%37) ve immobilizasyon (\%18.4) saptanmıştır. En az oral kontraseptif kullanımı (\%1.5) olup 10 hastada (\%7.5) predispozan faktör tespit edilememiştir. $\mathrm{Bu}$ bulguların literatürle uyumlu olduğu görüldü.

Uzamış immobilizasyon, bacak-kas pompasının efektif çalışmaması sonucunda venöz valf sinüslerinde kan akımının yavaşlamasına yol açarak esas mekanizmaya neden olur. VSM ve vena safena parvada (VSP) çok sayıda kapakçık bulunur. Kapakçıklar normalde tek yönlü çalışarak venöz akımın proksimale doğru olmasını sağlarlar. Venöz kapakçıklardaki yetersizliklere bağlı reflüde ven akımı gözlenebilir bir şekilde bozulur (19). Bunun sonucunda trombüs oluşumu kolaylaşır. Trombüs, akımın şeklinin değiştiği 
ve türbülansın olduğu venöz kapakçıkların iç kesimlerine (valv sinüsleri) yerleşim gösterir. Trombüs, büyüdüdükçe damar içindeki kan akımını engelleyerek diğer kapak veya venöz segmente ilerler. Kısaca trombüsün kontrolü veya sınırlanması, esas olarak kan akımına bağımlıdır (20). SVT masum bir hastalık gibi kabul edilse de ancak çalışmalarda SVT'nin DVT'ye ilerleyebileceği bildirilmiştir (21). Konumu nedeniyle, VSM veya SFB'nin SVT riskinin en yüksek olduğu lokalizasyon olduğu düşünülmektedir (22). VSM proksimali ve SFB düzeyinde olan reflü varlığında, ana femoral ven seviyesinin distalinde ve safenofemoral veya safenopopliteal kavşaklardan en az $1 \mathrm{~cm}$ uzakta herhangi bir derin venöz segmentte reflü varlığında ortak reflüye yol açtığı saptanmıştır $(23,24)$.

Vücuttaki en uzun venöz yapı olan VSM ve dalları yüzeyel venöz sistemin en önemli bileşenleridir. Fizyopatolojisinde akım değişiklikleri bulunan alt ekstremite venöz yetmezliği ve bunun neden olduğu varisler, en sık VSM'de (\%60), daha az olarak da VSP, perforan venler ve pelvik venlerden kaynaklanır (25). VSM, safen kompartmanı olarak tanımlanan, derindeki musküler fasya ile yüzeydeki safenöz fasya arasında yeralır. VSM safen kompartmanın dışarısında yüzeyel seyir gösterebilir. Bu seviyelerde destekleyici fasyanın olmaması, safenöz fasya yüzeyelindeki variköz yapılara zemin hazırlamaktadır (26).

RDUS incelemede VSM'nin tanınması için kullanılan "eye sign" (Egyptian eyes) ilk defa Bailly tarafindan tanımlanmıştır. "Safenöz kompartman" bulgusu VSM'yi tanımlamak için kullanılan ana kriterlerdir (27). VSM anatomisi oldukça değişkendir. Birçok varvasyonlar görülmektedir. Ancak \%50-55 hastada VSM anatomi kitaplarındaki seyri izler. Farklı oranlar olan çalışmalar da vardır. Varyasyonlar femoral ven ve VSP'de görülebilmektedir (28).

Çalışmamızda DVT olgularına yapılan RDUS inceleme raporlarında Ricci ve Caggiati sınıflamasına göre incelendi (14). Bu sinıflamaya göre en yaygın olarak, anatomi kitaplarında bahsedilen form olan A tipi 70 hastada (\%55.3) görüldü. En az rastlanılan formu ise 2 hastada (\%1.5) saptanan Tip B (VSM duplikasyonu) olmuştur. Bu bulguların literatüre uygun olduğu görüldü (29). Fakat VSM duplikasyonunun görülme sıklığı çalışmalarda \%1-35 arasında değişebilmektedir (30). Literatürdeki VSM duplikasyonun sık görülme sıklığını bildiren çalışmaların çoğunluğunda, muhtemelen vakaların birçoğu Ricci ve Caggiati'nin double sistem olarak adlandırdıkları olgular oluşturmakta ve bundan dolayı VSM'nin gerçek duplikasyon göstermediği durumlar VSM duplikasyonu olarak saptanmaktadır (31).

Ayrıca, 2014 yılında Oğuzkurt, venöz yetmezliği olan hastaları inceledi ve vakaların \%33'ünün, venöz yetmezliği olan veya olmayan hastaların benzer sıklıkta VSM varyasyonu gösterdiğini buldular (32).

Safen kompartmanına göre VSM lokalizasyonu açısından önemli bir fark olmadığını gösteren yukarda bahsedilen çalışmaların yanı sıra, Pittaluga ve Chastane venöz yetmezliği olan hastalardan 1.950 alt ekstremite incelendiğinde 299 (\%15.3) segmental VSM apilazisi vakası buldu. Safen kompartmanının venöz sisteme koruma sağladığını ve eksikliğinin venöz yetmezliğin hızlı ilerlemesi için önemli bir risk faktörü olduğunu varsaydilar (33).

2002'de Ricci ve Cavezzi bir çalışmalarında 493 bacağ araştırdı. Çalışmada VSM varyasyonları ile varis prevalansının ilişkili olduğunu bildirdiler. 2004 yılında Caggiati ve Mendoza, segmental hipoplazi oranının kontrol grubunda \%12 ve venöz yetmezliği olan hastalarda $\% 25$ olduğunu gösterdiler. Patofizyolojik bakış açısından VSM, VSM hipo/aplazisi olan vakalar safen bölmesi tarafindan desteklenmez. Aksesuar veya kol ven, yüzeysel bir konuma sahip ince duvarlı bir yapıya sahiptir ve çevresindeki kas hacmi küçüktür. Tüm bu faktörlerin varis gelişme riskini artırdığını öne sürmüşlerdir $(26,34)$. 
Bir çalışmada 65 SVT olan hastanın incelenmesinde \%24 hastada eşlik eden asemptomatik DVT saptanmıştır. SVT'nin hayatı tehdit etmediği düşünülse de DVT ile ilişkisinden dolayı dikkatli evalüasyonu önermişlerdir (35).

Başka bir çalışmada VSM varyasyonu olan hastalarda, dizin üzerinde hipoplastik segmentleri olan hastalarda deri değişiklikleri ve DVT anlamlı olarak daha sık görülürken, diz bölgesinde daralan segmentleri olan hastalarda venöz yetmezlik anlamlı olarak daha s1k olarak bildirilmiştir. Ayrıca aynı çalışmada $5 \mathrm{~cm}$ 'den uzun hipoplastik segmentleri olan hastalarda deri değişiklikleri ve komorbid DVT anlamlı olarak daha yaygın olduğunu gözlemişler (36).

Svetlana Jovic ve ark.'nın yaptıkları çalışmada, 97 hastada (\%53.89) alt ekstremitede yüzeysel damarları varyasyonsuz tipi (tipA), 83 hastada (\%46.11) çeşitli varyasyonlar saptamışlar. Alt ekstremite DVT'si yüzeysel venlerinin anatomik varyasyonları olan hastalarda \%50 daha çok olduğunu bildirmişler (37). Bunun yanında Simpson ve ark. yaptı̆̆ ekstremitede venlerinde varyasyon olan hastaların sadece \%0.4'ünde trombotik değişiklikler gördüklerini bildirmişlerdir (38).

$\mathrm{Bu}$ çalışmanın birinci kısıtlılığı retrospektif olmasıdır. İkinci kısıtlılığı olarak hasta sayısının az olması ve üçüncü olarak da daha hasta grubunun homojen olmamasidir.

Sonuç olarak; VSM varyasyonlarının DVT gelişmesi ile arasında bir ilişki bulunamamıştır. Çalışmadaki VSM varyasyonları normal popülasyonda görülen tipleriyle benzer oranda saptanmıştır.

Çatışma Beyanı: Çalışmada herhangi bir çıkar çatışması bulunmamaktadır.

Araştırmacıların Katkı Oranı Beyanı: Yazarlar makaleye eşit oranda katkı sağladıklarını beyan ederler. Destek / Teşekkür Beyanı: Çalışmada herhangi bir kişi ya da kuruluştan maddi destek alınmamıştır.
Etik Kurul Onamı: Kırıkkale Üniversitesi Girişimsel Olmayan Araştırmalar Etik Kurulu, tarih: 30.09.2020, say1 no: 2020.09 .10 .

\section{KAYNAKLAR}

1. Wallis M, Autar R. Deep vein thrombosis: clinical nursing management. Nurs Stand. 2001;15(18):4754.

2. Nutescu EA. Assessing, preventing, and treating venous thromboembolism: evidencebased approaches. Am J Health Syst Pharm. 2007;64(7):513.

3. Heit JA. The epidemiology of venous thromboembolism in the community. Arterioscler Thromb Vasc Biol. 2008;28(3):370-2.

4. Forster AJ, Wells PS. The rationale and evidence for the treatment of lower-extremity deep venous thrombosis with thrombolytic agents. Curr Opin Hematol. 2002;9(2):437-42.

5. Stamatakis JD, Kakkar VV, Sagar S, Lawrence D, Narrn D, Bentley PG. Femoral vein thrombosis and total hip replacement. Br Med J. 1977;2(6081):2235 .

6. Stone J, Hangge P, Albadawi H, Wallace A, Shamoun F, Knuttien MG et al. Deep vein thrombosis: pathogenesis, diagnosis, and medical management. Cardiovasc Diagn Ther. 2017;7(3):276-84

7. Labropoulos N, Delis K, Nicolaides AN, Leon M, Ramaswami G, Volteas N. The role of the distribution and anatomic extent of reflux in the development of signs and symptoms in chronic venous insufficiency. J Vasc Surg. 1996;23(5):50410.

8. Verlato F, Zucchetta P, Prandoni P, Camporese G, Marzola MC, Salmistraro G et al. An unexpectedly high rate of pulmonary embolism in patients with 
superficial thrombophlebitis of the thigh. J Vasc Surg. 1999;30(6):1113-15.

9. Lurie F. Venous haemodynamics: what we know and don't know. Phlebology. 2009;24(2):3-7.

10. Thomson H. The surgical anatomy of the superficial and perforating veins of the lower limb. Ann R Coll Surg Engl. 1979;61(7):198-205.

11. Somjen GM. Anatomy of the superficial venous system. Dermatol Surg. 1995;21(1):35-45.

12. Quenet S, Laporte S, Decousus H, Leizorovicz A, Epinat M, Mismetti $\mathrm{P}$ et al. Factors predictive of venous thrombotic complications in patients with isolated superficial vein thrombosis. J Vasc Surg. 2003;38(5):944-9.

13. Decousus H, Leizorovicz A. Superficial thrombophlebitis of the legs: still a lot to learn. J Thromb Haemost. 2005;3(6):1149-51.

14. Ricci S, Caggiati A. Echoanatomical patterns of the long saphenous vein in patients with primary varices and in healthy subjects. Phlebology. 1999;14(2):5460.

15. Di Nisio M, Van Es N, Büller HR. Deep vein thrombosis and pulmonary embolism. Lancet. 2016;388(10063):3060-73.

16. Kniffin WD Jr, Baron JA, Barrett J, Birkmeyer JD, Anderson FA Jr. The epidemiology of diagnosed pulmonary embolism and deep venous thrombosis in the elderly. Arch Intern Med. 1994;154(5):861-6.

17. Lee YK, Chung CY, Koo KH, Lee KM, Ji HM, Park MS. Conflict of interest in the assessment of thromboprophylaxis after total joint arthroplasty: a systematic review. J Bone Joint Surg Am. 2012;94(1):27-33.

18. Hopkins NF, Wolfe JH. ABC of vascular diseases. Deep venous insufficiency and occlusion. BMJ, 1992;304(6819):107-10.

19. Mihmanlı İ. Vasküler Ultrasona Giriş. Birinci baskı, İstanbul. İstanbul Medikal Yayıncılık, 2006.
20. Naidich JB, Feinberg AW, Karp-Harman H, Karmel MI, Tyma CG, Stein HL. Contrast venography: reassessment of its role. Radiology. 1988;168(1):97100.

21. Bounameaux H, Reber-Wasem MA. Superficial thrombophlebitis and deep vein thrombosis. A controversial association. Arch Intern Med. 1997;157(16):1822-4.

22. Blumenberg RM, Barton E, Gelfand ML, Skudder P, Brennan J. Occult deep venous thrombosis complicating superficial thrombophlebitis. J Vasc Surg. 1998;27(2):338- 43.

23. Kostas T, Ioannou C, Touloupakis E, Daskalaki E, Giannoukas AD, Tsetis D et al. Recurrent varicose veins after surgery: a new appraisal of a common and complex problem in vascular surgery. Eur J Vasc Endovasc Surg. 2004;27(7):275-82.

24. Somjen GM, Royle JP, Fell G, Roberts AK, Hoare MC, Tong Y. Venous reflux patterns in the popliteal fossa. J Cardiovasc Surg. 1992;33(4):85-91.

25. Brasic N, Lopresti D, McSwain H. Endovenous laser ablation and sclerotherapy for treatment of varicose veins. Semin Cutan Med Surg. 2008;27(9):264-75.

26. Ricci S, Cavezzi A. Echo-anatomy of long saphenous vein in the knee region: proposal for a classification in five anatomical patterns. Phlebology. 2002;16(3):111-9.

27. Bailly M. Cartographie CHIVA. In: Elsevier Masson SAS, ed. Encyclopedie Medico-Chirurgicale. $1^{\text {st }}$ ed. Paris. Editions Techniques, 1993:43-161.

28. Caggiati A, Bergan JJ, Gloviczki P, Jantet G, Wendell-Smith CP, Partsch H. Nomenclature of the veins of the lower limbs:an international interdisciplinary consensus statement. J Vasc Surg. 2002;36(2):416-22.

29. Yuce I, Oguzkurt L, Eren S, Levent A, Kantarci M, Yalçın A et al. Assessment of posterior accessory great saphenous vein of the leg using 
ultrasonography: a preliminary study. Surg Radiol Anat. 2016;38(5):123-9.

30. Kockaert M, De Roos KP, Van Dijk L, Nijsten T, Neumann M. Duplication of the great saphenous vein: a definition problem and implications for therapy. Dermatol Surg. 2012;38(6):77-82.

31. Ricci S, Caggiati A. Does a double long saphenous vein exist? Phelebology. 1999;14(5):59-64.

32. Oguzkurt L. Ultrasonography study on the segmental aplasia of the great saphenous vein. Phlebology. 2014;29(9):447-55.

33. Pittaluga P, Chastanet DS. Influence on chronic venous insufficiency of primary absence of the great saphenous vein in the saphenous compartment at the thigh. J Vasc Surg Venous Lymphat Disord. 2013;1(1):101.

34. Caggiati A, Mendoza E. Segmental hypoplasia of the great saphenous vein and varicose disease. Eur J Vasc Endovasc Surg. 2004;28(8):257-63.

35. Binder B, Lackner HK, Salmhofer W, Kroemer S, Custovic J, Hofmann-Wellenhof R. Association between superficial vein thrombosis and deep vein thrombosis of the lower extremities. Arch Dermatol. 2009;145(7):753-7.

36. Sipahi M, Bolat A, Serin HI, Erkoc MF, Acikgoz B, Kulah B. Clinical Manifestations in Patients with Segmental Hypoplasia of Great Saphenous Vein. Kuwart Medical Journal. 2017;49(2):119-23.

37. Jovic S, Delic J, Ljuca F, Mujanovic E, CustendilDelic S, Zabic A et al. Frequency of superficial and deep vein thrombosis in patients with variations of superficial veins of lower extremities. Med Arh. 2012;66(1):16-8.

38. Simpson WL, Krakowski DM. Prevalence of lower extremity venous duplication. Indian JR Radio Imaging. 2010;20(3):230-4. 